\title{
The Role of Aesthetic Style in Alleviating Anxiety About the Future
}

\author{
James Carney
}

Style is a form of concrete metaphysics. It is metaphysical to the extent that every given style implies that all of reality has a particular character; it is concrete because it structures the materials from which representations of actual or possible states of affairs are made. Moreover, style is a cultural universal: every culture on record has stylistic traditions, and even the refusal of style in favor of (purported) direct representation is itself a stylistic choice (Barthes, 2000; Dutton, 2009). For these reasons, style and its associated phenomena are crucially important to the understanding of human culture. And yet, while there is no shortage of historical expositions on particular styles of representation, the origin of style itself is far less frequently theoriszd. Even where worthwhile expositions do exist (e.g., Meyer, 1987; Schapiro, 1997), they tend to focus on specific types of style, such as musical or pictorial, at the expense of a more general appreciation of the features common to all styles.

In the present chapter, I shall present a new account of style that attends to the wider principles underwriting it. My goal will be to show that the purpose of style is to establish a cognitive orientation towards the future - that is, it represents the world as being inherently more predictable. In doing this, I argue that style succeeds in reducing anxiety with respect to the future by framing certain aspects of the future as probable and framing other aspects as less probable. I shall pursue this idea by way of concepts from information theory, which provide a powerful framework for dealing with symbolic systems from a quantitative perspective. Specifically, I shall show that style results from an agent's desire to establish an optimum balance between the simplicity of a model of the environment (technically, its entropy) and the model's accuracy. I hypothesize that the agent preserves low model entropy by

This research was supported by a fellowship from the Wellcome Trust awarded to the author, grant number 205493/Z/19/A.

\footnotetext{
J. Carney ( $ه)$

Brunel University London, London, UK

e-mail: James.Carney@brunel.ac.uk
} 
actively saturating the environment with model evidence, and thus preserves model integrity for longer by reducing prediction errors. This reduces anxiety by creating plausible expectations about future perceptual and cognitive experiences. Given that the accuracy of environmental models is subject to direct and indirect selection processes, my account of style will in the final account be evolutionary. However, I shall also show that the process of stylistic creation and elaboration is historically conditioned, and it is only when evolutionary processes are considered in conjunction with historical ones that any new knowledge about a particular style emerges.

I shall begin my account by summarizing our intuitions about style and how it has previously been theorized. As I do not aim to present a historical survey of thinking about style, this summary will not be exhaustive; nevertheless, it should map some general contours of the phenomenon. The next step will come with an introduction to some information-theoretic concepts, and how they relate to perception. This will be followed by the central part of my exposition, which connects perception, prediction, and style. I shall finish by showing how some of the features of style outlined in the first section can be explained and making some further predictions that invite testing.

\section{What Is Style?}

To begin, it is a useful exercise to summarize our intuitions about style. Though this may lack empirical rigor, it will at least provide a starting point for the formalization of the general principles at work in stylistic representation. Equally, it will allow for engagement to be made with some of the existing literature on the nature of style. For the record, I shall mostly use pictorial art for the purposes of example throughout the discussion: this should not be taken to imply that my conclusions only apply to the visual arts-in principle they have relevance for any medium, including language.

The most basic understanding of the concept of style identifies it as a principle of organization based on normative rather than practical precepts. Significantly, this principle is not co-extensive with the habits or heuristics of an individual agent. Though individuals certainly have stylistic signatures ('Kafkaesque'), their stylistic practices can vary over their lifetime ('Picasso's late style'); moreover, stylistic similarity is used to demarcate both collective aesthetic movements ('Russian suprematism') and longer periods of artistic or decorative production ('the medieval aesthetic'). Even more reductively, style can sometimes be used to capture the aesthetic and decorative norms associated with a cultural group, whether based on national origin ('French chic'), economic class ('bourgeois art'), or religious denomination ('Catholic expressiveness'). What this serves to show is that style has no obvious subject; it is a practice that can be implemented across multiple agents who are often distributed in time and space. Moreover, these agents need not even be compositionally human: deep learning algorithms have already produced visual and linguistic artefacts that are indistinguishable from human productions (Gatys, 
Ecker, \& Bethge, 2015; Gwern, 2019), and there is ample evidence of stylistic variation in animal communication (Botero, Pen, Komdeur, \& Weissing, 2010).

Clearly, then, any appreciation of style cannot begin with its cognitive implementation in humans; instead, it needs to identify the underlying structure that is common to its implementation (psychological or otherwise) across all subjects. In this regard, Meyer (1987) offers a useful starting point when he defines style as 'a replication of patterning ... that results from a series of choices made within some set of constraints' (p. 21) -with 'choice' to be understood in the weak sense as merely meaning that alternative patterns are possible. The value of this formulation is that it captures two components-contingency and necessity-that are visible in any coherent manifestation of style. The necessity derives from the fact that, at any point, a style will prohibit certain aesthetic choices being made; the contingency lies in the liberty of the subject to choose freely once these prohibitions are respected. Inevitably, matters are not so simple in reality, as styles typically evolve by permitting and incorporating small deviations relative to established norms (Adorno, 2004; Eco, 1985); equally, there is always the degree to which an artefact evinces a particular style (Munro, 1946). But even allowing these caveats, it remains the case that an aesthetic style - whether lodged in an individual, a genre, a movement, or a historical period - defines a set of normative standards that both positively and negatively select for aesthetic choices.

This issue of normativity brings to the fore another core feature of style-its polemical character. Friedrich Nietzsche (1927) captures one aspect of this in his discussion of the dichotomy, in Greek art, between the integrative, rationalist 'Apollonian' aesthetic and the disintegrative, hedonic 'Dionysian' aesthetic. One need not subscribe to Nietzsche's categories to appreciate the wider point: styles are defined by what they are not as much by what they are, so no style can exist without at least implicitly defining itself against a counter-style. Moreover, given that the subjects of a style are often (but not always) social collectives, this means that stylistic oppositions can be coordinated with political ones. This can be seen readily enough in the culture of the manifesto and the radical break, which often attends the emergence of a new style (Danchev, 2011). Equally, the stylistic innovations associated with marginal subcultures can be interpreted as implicit refusals of a dominant or received pattern of meanings. In Dick Hebidge's words (1979), these innovations "carry "secret" meanings which express, in code, a form of resistance to the order which guarantees their continued subordination' (p. 18). It takes no great conceptual leap from here to identify a style as an expression of a worldview-a claim we shall return to; for now, the idea to retain is that styles are typically (though not necessarily always) antagonistic with respect to each other.

A third feature that is visible in our intuitions about style is that individual styles are transitory. Depending on context, the degree to which this is so can vary. More strictly decorative expressions of style (such as found in fashion) have their value defined, at least in part, by novelty, and thus evince the transitory nature of style with particular effect. Equally, fast-moving milieux like the twentieth-century avant-garde, where unprecedented social and technological change was matched by equivalent innovation on the aesthetic sphere, are characterized by quick 
successions of different stylistic practices. (Indeed, some authors go so far as to claim that this drive for novelty will ultimately result in incoherent artforms [Martindale, 1990; Martindale \& Locher, 2009].) However, no human society is without history, and even relatively static societies-such as, presumably, those found in the Palaeolithic_-still see stylistic variation in time and space (Pigeaud, 2007). By the same token, even where styles persist over long durations, it remains the case that sub-styles and local variations proliferate, sometimes to the point of becoming distinct styles in their own right-a pattern most visible in the evolution of popular genres like science fiction and fantasy. All of this suggests that whatever function style may serve, it can only do so for a limited time: whether in the long or the short term, styles will eventually become exhausted, superannuated, or otherwise obsolete.

These, then, are some of the core intuitions that any theory of style should be able to reproduce. Before advancing to such a theory, however, it is important to address some possible objections to the wider project I am proposing. The most important of these centers on the viability of style itself as an analytical category. Such objections generally maintain that style has no objective reality and exists only as a denotative shorthand for historical particulars that cannot be meaningfully discussed outside of the material and contextual details of these particulars (Alpers, 1987; Smith, 1980). In practice, this results in a rejection of any general theory of style: 'it is because style is a historical phenomenon that an absolute science of style is impossible' (Jameson, 1972, p. 335). As such claims are so visibly the product of a politically aggressive historicism, it is tempting to just not bother engaging with them at all. However, a rebuttal is easily formulated. If the idea of style is to be rejected because it is abstracted from contingent material phenomena, then so too must concepts like species, gene, function, adaptation and many others that make up the lexicon of scientific biology. Biological forms, no less than cultural ones, are contingent, with the only difference being that the contingency lies in the operations of natural selection over any purported historical dialectic. While there is no shortage of theorists - past and present—who remain willing to intrude their political convictions into the natural order (Kolchinsky, Kutschera, Hossfeld, \& Levit, 2017), the utter failure of such approaches to create new knowledge presents its own verdict on their viability. For a concept like style, which is vastly simpler than even the most rudimentary biological system, it therefore follows that formalism is not only allowed, but mandated.

\section{Perception and Information}

Translating the features of style into a model requires formalizing them in an appropriate way. In this regard, the first step comes with taking a step back and making some observations concerning human perceptual experience and how it informs models of the environment. Once this has been done, it will become possible to mathematically model style as an attempt to reduce the information-processing 
demands of psycho-perceptual interaction with the world. Though this will lead the discussion into some mathematical formalism, I submit that this formalism will repay its presence by sharpening our understanding of how style works, and where it sits in relation to the wider repertoire of human activities.

Though perception is one of the largest research areas in psychology, it is also one of the most mature, with a core discovery being that human perception is not experienced as a continuous set of gradations; instead, continuous sensory inputs are discretized (often recursively) into perceptual elements (Marr, 1982, 2010; Nakayama, Motoyoshi, \& Sato, 2018; Sims, 2016). Moreover, these elements are not experienced in a neutral fashion. As explored in the enactivist literature, perceptual elements are at once registrations of the state of the world and invitations to act on it (Bach-y-Rita \& Kercel, 2003; Georgeon, Marshall, \& Manzotti, 2013). In the simplest case, these perceptual experiences center on spatial location and physical manipulation; more complex experiences-like those supported by cultural objects and experiences - are freighted with the sophisticated meanings and affordances that inform human intersubjective existence (Durt, 2017; Gallagher, 2008).

Putting this on a probabilistic footing, what emerges is that a human perceptual environment can be modelled as a set of $n$ discrete elements, $S$, such that each $s_{i} \in S$ for $1 \leq i \leq n$ picks out one of these elements. Necessarily, the full repertoire of these elements must be purely theoretical, as no analysis can ever enumerate them in advance.

$$
S=\left\{s_{1}, s_{2}, \ldots, s_{n}\right\}
$$

The value of this formalism is that a given perceptual environment, $S_{j}$ can then be defined by a probability distribution, $P_{j}$, where $p_{j i}$ denotes the probability of a randomly selected perceptual element in that environment being the element $s_{j i}$. In this scenario, each $p_{j i}$ for $1 \leq i \leq n$ can theoretically take a value between 0 and 1 inclusive, and the entire distribution sums to 1 - even if in practice there will always be some non-zero probability assigned to every element, though it might be vanishingly small.

$$
P_{j}=\left(p_{j 1}, p_{j 2}, \ldots, p_{j n}\right)
$$

More generally, what each $P_{j}$ here represents is a model of an environment. By defining a set of expectations, this model has an obvious role in guiding the allocation of resources (including attention) for an organism or process embedded in the given environment (Clark, 2016). As the environment varies, so too will the model, with both the duration (a moment, a day, a lifetime) and the scope (an organ, a body, a landscape, a cosmos) of the model being parameters of variation. For this model to be useful, a necessary (but not sufficient) condition is that it has low entropy. As the logic behind this constraint may not be familiar to some readers, I will now briefly outline the concept of entropy (technically, Shannon entropy) as it functions in information theory, given that this is crucial to the discussion that follows. 
On one definition, entropy is 'a measure of the information contained in a message as opposed to the part of the message that is strictly determined (hence predictable) by inherent structures' (Kuzemsky, 2017, p. 275). That is to say, entropy measures how unpredictable a message is, with messages high in entropy being unpredictable and those low in entropy being predictable (Cover \& Thomas, 2005; Shannon, 1948). As the core concept of information theory, entropy allows for symbolic complexity to be quantified. This is done by way of the probability distribution of symbols in a message, such that the entropy, $H$, of a distribution, $X$, is defined as the product of the probability of a symbol occurring and the logarithm of this probability, summed across all symbols $x_{i} \in\left\{x_{1}, x_{2}, \ldots, x_{n}\right\}$. When the base of the logarithm is 2 , the outcome is measured in bits.

$$
H(X)=-\sum_{i} p\left(x_{i}\right) \log _{2} p\left(x_{i}\right)
$$

The mathematical details here are less important than the intuition behind them, which is that entropy is highest for systems with several equiprobable outcomes. Take the entropy for a coin toss with a coin that has an equal chance of heads or tails coming up (i.e. $p(H)=1 / 2 ; p(T)=1 / 2$ ). The entropy of this system can be calculated as follows:

$$
-1 \times\left[\left(\frac{1}{2} \times-1\right)+\left(\frac{1}{2} \times-1\right)\right]=1
$$

This shows that only one bit of information is needed to encode the states of this system. Very different results emerge when the coin is biased. Figure 1 shows the effect of shifting the bias from one extreme ( $H$ always comes up, or $p(H)=1)$ to the other ( $T$ always comes up, or $p(T)=1$ ). As can be seen, entropy is zero for the extreme scenarios as the system is perfectly predictable and there is no uncertainty; as calculated above, the maximum entropy occurs when $H$ and $T$ occur with equal probability.

Bringing this back to the discussion of perceptual models, it should now be clearer why the model $P_{j}$ should have low entropy if it is to be effective. A model that defines a uniform probability distribution cannot be used to create a differentiated set of expectations, because all outcomes are expected equally. Far more useful is a model that accurately identifies specific outcomes as being more likely than others in a given perceptual environment; better yet is a suite of models that recalibrate expectations across different scenarios. If this so, then it is likely that there should be an evolutionary selection pressure for accurate, low entropy models - and this, indeed, is what we see in the literature linking anxiety to uncertainty. Here, the suggestion is that the experience of anxiety can be understood as a penalty function for cognitive models of the environment that fail to offer identifiable predictions (Craske, 2003; Craske et al., 2009; Ellsberg, 1961; Hirsh, Mar, \& Peterson, 2012; Lake \& LaBar, 2011; Sullivan, 2016). Though such evidence is only phenomenologically accessible with respect to humans (through self-report), there is evidence 


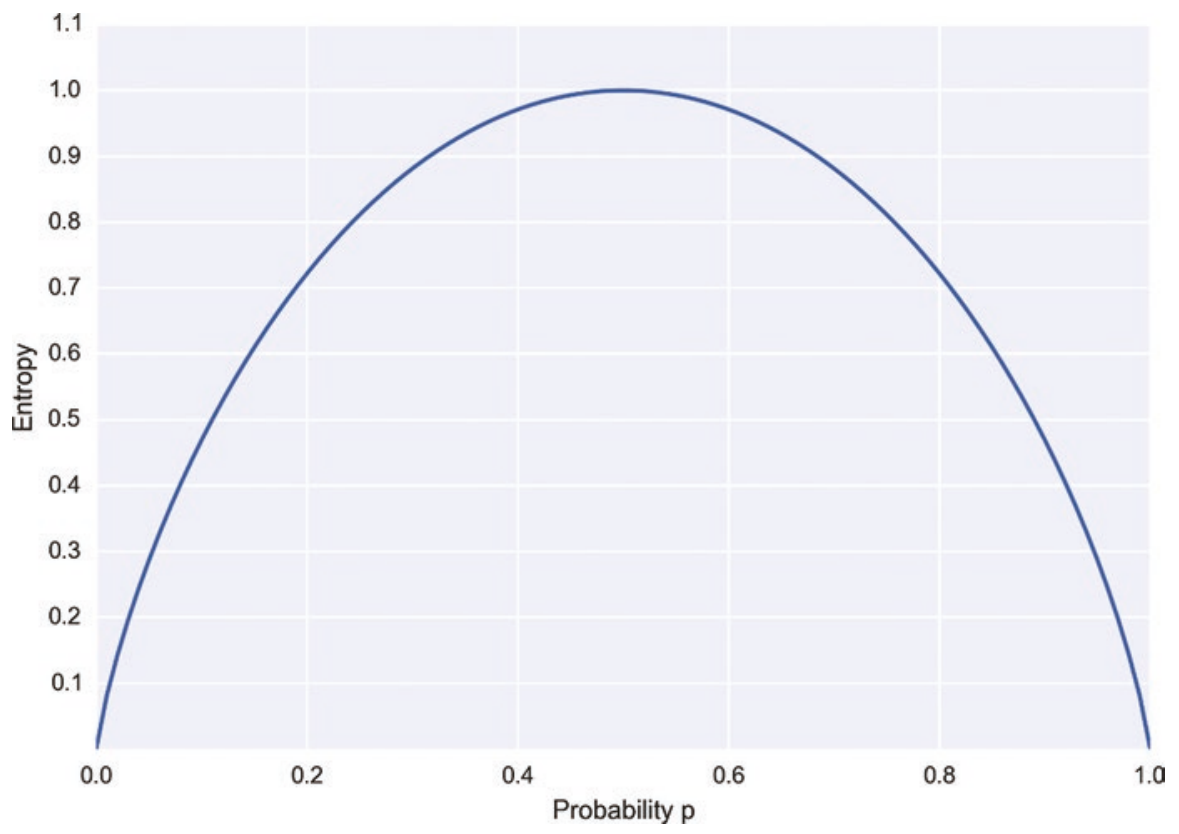

Fig. 1 Entropy of a coin toss for $p(H)=p$ and $p(T)=1-p$ as $p$ takes values in the $[0,1]$ interval. Note that entropy is at a maximum for an unbiased coin toss, when both $p(H)$ and $p(T)=0.5$

from neuroscience that it is present in animals, too (e.g., Grillon, Baas, Lissek, Smith, \& Milstein, 2004; Herry et al., 2007). Thus, one of the central aversive sensations for an organic agent is linked directly to the entropy of its model of the environment. In such a scenario, the clear incentive that emerges is for the agent to reduce anxiety by optimizing for low-entropy models.

And it is here that the problem emerges. Building a predictive model of the environment is extremely difficult, largely because discrete perceptual elements admit being combined into composites that are themselves experienced discretely. The result of this combinatorial facility is the quick supervening of distributions that are intractable with respect to computing probabilities. To see this, imagine a highly parsimonious ontology of 100 colors; at the same time, allow that a discrete experience can consist of combinations of up to four colors together. The approximately four million potential perceptual items that emerge from this is already too large for any on-the-fly cognitive system to easily deal with - a situation that becomes entirely unworkable for systems with anything approaching real-world complexity. Necessarily, this latter scenario will expose an organism to all the existential threats that attend a poor environmental model, which will ultimately result in those organisms with more effective models being genetically selected for. The result is to trap the agent between two competing imperatives: the desire to avoid anxiety by optimizing for simple, low-entropy models of the world, and the difficulty of satisfying 
model accuracy due to a combinatorial explosion in the number of possible elements of experience.

A useful way to quantify this situation is by way of what are known as Bregman divergences (Bregman, 1967). These divergences measure the difference between a 'true' distribution, $P$, and an estimating distribution, $Q$. As such, they provide a good metric of how well a model fits the environment. For the purposes of illustration, the most useful of these is the Kullback-Leibler divergence-also known as the relative entropy-between two distributions (Kullback \& Leibler, 1951). This measures how much extra information, in bits, is needed to model a distribution $P(X)$ when an approximating distribution $Q(X)$ is already known.

$$
D_{\mathrm{KL}}(P \| Q)=\sum_{x \in X} P(x) \log _{2} \frac{P(x)}{Q(x)}
$$

When the distributions are identical, the divergence is zero, and no extra information is needed to represent $P$ in terms of $Q$; as $D_{\mathrm{KL}}$ increases, the approximating distribution becomes less and less accurate as a model of the target distribution. In the context under discussion, this means that the need for accuracy will drive organisms to create models of the world that drive $D_{\mathrm{KL}}$ down; however, the impossibility of doing so will — through the mechanism of anxiety - push organisms towards lowentropy approximations that can often drive $D_{\mathrm{KL}}$ up. How can this dilemma be resolved?

It is here, after our information-theoretic detour, that it becomes possible to return to the topic of style. As we shall now see, style can be theorized as an attempt to inject maximum predictability into the environment, subject to constraints of model accuracy. What this should show is that style is a local example of a general tendency on the part of organisms to optimize their behavior by minimizing prediction error while maximizing model simplicity.

\section{Style and Prediction}

Every style is encountered as an arrangement of the material world-whether that be in the form of paint on a canvas, patterns of sound in the air, or the movement of limbs on the human body. As such, each style exists as part of a perceptual repertoire. Given this, the perceptual considerations outlined in the previous section also apply to style. In fact, their application is even more appropriate. For the perceptual elements circumscribed by a particular style are always exaggerated-one of the functions of a style is to draw attention to itself (Shklovsky, 1965). This is a logical prerequisite, to the extent that a style cannot be individuated from a background unless it is perceptually salient; however, neuroscientific research also shows that aesthetic phenomena have distinctive, measurable impacts on neural processing (Ishizu \& Zeki, 2013; Seeley, 2013). In this sense, style offers a condensed form of 
perception, such that a stylized element is freighted with greater salience than a non-stylized element.

Paralleling this exaggeration of perceptual salience, most styles are enacted across a smaller number of perceptual elements than are likely to comprise the world of phenomenal experience. Take De Stijl, the aesthetic style associated with Piet Mondrian and other Dutch artists of the inter-war period. In Mondrian's words, this aesthetic ignores 'the particulars of appearance, that is to say, natural form and color. On the contrary it should find its expression in the abstraction of form and color, that is to say, in the straight line and the clearly defined primary colour' (qtd. in Huntsman, 2016, p. 141). Thus, in this aesthetic, there are essentially two elements: the straight line and the set of primary colors. This is a generative system, so there are certainly more than two perceptual experiences available through it; nevertheless, the probability distribution, $P$, defined across the set of possible perceptual elements, $S$, will, in this instance, consist mostly of zeroes, with those elements that are not zero having a high value. Naturally, a more mimetically oriented style than De Stijl will emphasize a greater number of elements, but any style that falls short of a one-one reproduction of reality—which is to say, every style-will still perform this reductive operation.

What all of this points to is the functional role of style in making an environment more predictable. Because each style is associated with a set of perceptual expectations, any environment that is saturated with that style lends itself to being predicted with greater facility than an environment which is not. Thus, if an agent wishes to reduce the uncertainty in the environment, it can do so by actively shaping the environment in accordance with the precepts of a given style. In this way, 'action then ensures that prior expectations are met and desired states are frequented' (Friston, 2009 , p. 299). If this perspective is allowed, style becomes much more than mere decoration; it is instead the efferent arm of a process of uncertainty reduction, where the environment is actively manipulated so as to render it consistent with a set of model expectations. Intuitive support for this idea comes from the observation that a core impulse of a style is to percolate outwards. What began as niche aesthetic in Dutch abstract art now defines the perceptual experience of the London underground (Lloyd, 2017), just as the discordant aesthetic of punk music projected itself into the jarring sartorial choices of its performers and followers (Hebidge, 1979). The examples could be multiplied, but the point remains the same: styles colonize environments, thereby making them more predictable.

The first impact of this colonization will be to reduce anxiety. If high-entropy scenarios are intrinsically more anxiety-provoking, the saturation of the environment with predictable stylistic features will attenuate the baseline uncertainty that attends perception and action. While this claim is difficult to test empirically, the evidence that does exist supports it. For instance, there is a robust literature on the therapeutic effects of artistic creation on the experience of anxiety (Curry \& Kasser, 2005; Jang, Kang, Lee, \& Lee, 2016; Sandmire, Gorham, Rankin, \& Grimm, 2012; Slaytohn, D’Archer, \& Kaplan, 2010; van der Vennet \& Serice, 2012). So also, conditions like autism spectrum disorder that may derive from impaired predictive abilities are often attended by repetitive 'stimming' (self-stimulation) and 
pattern-matching behaviors that regularize the environment by actively making it more predictable (Lawson, Rees, \& Friston, 2014; Sinha et al., 2014). While it would be unwise to generalize these observations across the entire cultural record, the fact remains that they provide a compelling explanation for the excess that characterizes style. As has been observed by several commentators, aesthetic stylization is something of an evolutionary mystery: though consuming of resources, aesthetic phenomena seem to offer no functional advantages (Dutton, 2009; Pinker, 2007; Tooby \& Cosmides, 2001). On the view offered here, the impulse to stylization is to be found in the manipulation of the environment so as to reduce the likelihood of encountering surprising states; the seeming superfluity of stylization derives from the requirement that this must be done at scale if it is to be effective.

But if this satisfies one of the constraints identified of model building-the reduction of entropy-it leaves to one side the other constraint, which centers on model accuracy. This, it will be remembered, concerns the combinatorial explosion in the possible forms of perceptual experience on the back of linear increases in the elements that compose these experiences. If the model of style offered here has any value, then this pressure should be in some way visible in how style manifests itself in the cultural record. And this, in fact, is the case, to the extent that the duration of a style is intrinsically related to the accuracy of the model of the environment it represents.

To see how this is so, we need to look at the items from which a style is composed. Though these items can, in principle, be anything, it is notable that the duration of a style is to an important degree determined by the psychological, cultural, and historical significance of the items that compose it. 'Significance' can, of course, mean anything, so it is as well to be clear and indicate that its meaning here centers on what might be thought of as the explanatory salience of an item. In lived experience, there will always be some class of forms that seem expressive of a deeper or hidden principle of structure or value. Sometimes, these will coincide with biological cues, which evolution has conferred with a suggestive or stimulating power (Dawkins \& Krebs, 1979; Thom, 1990)—such forms are visible, for example, in the big-breasted Venus figurines of the Palaeolithic (Schebesch, 2013). Other times, it may be that the frequency with which a specific form is encountered increases, due to material changes in the environment-in the way, for instance, that the new technologies of the early Bronze Age served to make perceptual forms associated with metal smelting more culturally salient (Brück, 2006; Sørensen \& Rebay, 2008). In both cases, the relevant forms point to a set of hidden variables or propositions, $Z$, that stand behind the flux of phenomenal experience (e.g. 'the world is/is not the creation of a benign mother'; 'fire is/is not the underlying nature of reality'). Necessarily, these purported hidden variables will enter into cosmological and philosophical speculation on the nature of reality (or some part of it), in that they will define a probability distribution, $P(Z)$, that functions as an interpretive prior in the assessment of new sense data. To greater and lesser degrees, the encounter with real-world evidence, $X$, will change the estimation of the prior probabilities assigned to the items of the $P(Z)$ distribution in accordance with Bayes' formula. 


$$
P(Z \mid X)=\frac{P(X \mid Z) P(Z)}{P(X)}
$$

That is, corroborating or disconfirming evidence will be used to update the probabilities assigned to the items that comprise $Z$ ('given evidence $X$, it is more/less likely that the world is/is not the creation of a benign mother'). If model accuracy is a criterion of interest, then these hidden variables - if actually present-will therefore allow for low entropy to be coordinated with high accuracy. The question is, are they present?

It is here that the issue of stylistic duration comes into view. As part of the perceptual environment, the elements of a given style will act as evidence for the likelihood of an associated set of hidden variables - whether through causal implication, symbolic association, or subjective conviction (Peirce, 1958). However, when a style is the only evidence for these variables that are encountered, the reduction in anxiety is offset by the loss of accuracy. In this scenario, an agent can either increase evidence by further saturating the environment with stylistic elements or else abandon the style entirely. Conversely, when a style aligns with other aspects of the environment, it adds further evidence for a posited set of hidden variables; here, the style is likely to persist due to satisfying both the low entropy and high accuracy constraints. Thus, the duration of a style is directly informed by the set of hidden variables that motivate it.

This hypothesis predicts several known categories of style. At one extreme, there is the $\mathrm{fad}$, where the hidden variables offer some contingent or facile model of the world that cannot be sustained; at the other extreme, a style becomes a tradition, where the hidden variables correspond to cosmological or metaphysical principles that cannot easily be disproved. The only other possibility-the hidden variables exist and perform genuine explanatory work-is almost never encountered in stylistic elaboration, as it is epistemologically equivalent to scientific theorizing. The one exception (to the author's knowledge) is the set of action roles found in narrative, to the extent that these provide folk-psychological ways of understanding social behavior that actually reflects the structure of human motivations (Gallagher, 2011; Hutto, 2009). For reasons we explore in Carney, Robertson, and Dávid-Barrett (2019), this produces a situation in which the hidden variables come to be taken as an approximation of reality without needing to be supported by conditioning evidence. (That is, they make up the estimating distribution in a process of variational Bayesian inference, where the estimated distribution of motives is intractable due to the combinatorial explosion, as groups get bigger, in representations of the form ' $\mathrm{I}$ believe that $A$ thinks that $B$ is certain that $D$ knows ... that $X$ is the case'.) But leaving aside the special case of narrative, what emerges is that model accuracy is the key variable that impacts on the duration of a style. When the hidden values that inform a style perform no explanatory work, the style is evanescent (if potentially intense); when the style evokes variables that are less easily dismissed, it will be compelling for much longer. In situations where there is perfect predictive knowledge, I suggest there will be no style at all. However, I caveat this by suggesting that 
even a perfect model can only predict accurately with exhaustive data, and for most situations, we do not have (and probably never will have) exhaustive data. We can aspire to successful knowledge about physical processes, and to the extent that we do, we no longer stylize these processes because our uncertainty is reduced. Against this, there is no scientific theory that usefully accounts for dealing with social situations and uncertainties at the level they are experienced, because there are no nontrivial scientific laws predicting the exact form my dinner party, night on the town, and first encounter with my in-laws will take.

As these considerations are somewhat abstract, it is useful to close this section with an example - once again, De Stijl. When De Stijl emerged, the Netherlands was undergoing a wave of industrialization that consolidated its position as 'part of the advanced economic core of North-West Europe' (Wintle, 2000, p. 245). At the same time, massive and unprecedented infrastructural projects like the reclamation of land from the Zuidersee were being initiated (Van Lier \& Steiner, 1982). When combined with international developments in physics and mathematics, it is easy to see how such a milieu would incubate a model of the world as intrinsically ordered and rational. At a perceptual level, this would be experienced by way of increased encounters with manufactured items and landscapes, where the idiosyncrasies of natural and artisanal forms are replaced by a geometrical schematism composed of straight lines, right angles, and formal equivalences between objects. Though familiarity makes these forms unexceptionable to present-day eyes, at the time they would have been salient as indicators of a rationalist, collective-and, in the USSR of the same period, revolutionary-re-ordering of the human lifeworld. This in turn would have facilitated a world-model that has, as its animating claim, the rejection of subjectivity as a generative principle and the embracing of impersonal processes. It is not surprising, therefore, to see this given expression in the first manifesto of $D e$ Stijl, which juxtaposes the subjectivist impulse of earlier art-'traditions, dogmas, and the predominance of the individual'-against the 'relationship of equality between the universal and the individual' that attends the new style (Conrads, 1970, p. 39). Thus, the De Stijl aesthetic — and indeed the modernist aesthetic more generally_can be understood as an attempt to infuse the environment with perceptual evidence for a low-entropy model of the world that retains a high accuracy value due to the historical salience of the perceptual elements that compose it.

\section{Conclusion: Some Predictions}

Style reduces anxiety about the future by making the environment more predictable, subject to the constraints of model accuracy-with environment corresponding to both the specific field of aesthetic creation and the more general world of all fabricated objects and processes. That, at least, is the claim I am making here. If correct, this model of style should both account for our existing intuitions and-ideallyallow for new insights. We have already seen how the transitory nature of style is explained by the model accuracy parameter. Stylistic forms that do not evince 
hidden explanatory variables (or do so implausibly) are evanescent; styles that point to hidden variables less easy to disprove tend to last longer. Explanations are also available for the normative and polemical character of style. Specifically, styles are normative and polemical for the same reason: each one defines a model of the world, and models of the world are exclusive (mathematically, the probability distribution across the elements of the model sums to one). This means that no style can accommodate the prescriptions of another without radically changing its own forms and becoming a new style. At the same time, the political function of a worldview that is encoded in a set of stylistic forms cannot admit another style without conceding legitimacy. While this latter point does not mean that syncretic styles are impossible, it does suggest that they will be most common (like religious syncretism) in times when a secure collective identity is available. Compare, for instance, the openness of the later Roman Empire to regional aesthetics with the competitive elaboration of highly individual styles by small tribal societies that exist in close proximity to each other (e.g. in Papua and West Papua).

But the question remains: What are the new insights provided by the informationtheoretic model? We have already seen how varying the accuracy parameter generates predictions; it is perhaps worth finishing by cataloguing the predictions that follow from varying the simplicity parameter. In practice, this means varying the entropy of the model associated with the style. Doing this at once introduces considerations of cognitive load. All styles are simplifications, but some styles simplify to a far greater degree than others - and because these styles are predictable, they impose lower demands on working memory and attention (Carney, 2019; Hunter \& Pisoni, 2018). This contrasts with more elaborate or ornate styles, which impose high demands by approximating more and more to the degree of complexity that attends real-world phenomena. From this, two predictions emerge. The first is that stable, unchanging contexts will support high-entropy styles, whereas unstable contexts will support low-entropy ones. The second is that the greater the psychological distance associated with a topic of aesthetic representation, the lower the entropy of the associated style is likely to be. ('Distance', in this instance, picks out displacement with respect to space, time, familiarity, and likelihood—see below.)

With respect to the first prediction, unstable environments place obvious demands on cognitive budgets with respect to time, energy, memory, and attention (Dunbar, Lehmann, Korstjens, \& Gowlett, 2014; Mithen, 1995; Stout, Hecht, Khreisheh, Bradley, \& Chaminade, 2015). Any activity that competes for the same resources will, therefore, negatively impact on the processing of threats and opportunities by reducing these resources, and thereby raise anxiety. Conversely, environments that are highly predictable can free up cognitive resources for activities that are not directly adaptive without compromising agent survival. Thus, the simplicity parameter-the model entropy-associated with anxiety reduction in style will have a much tighter bound in challenging environments. In practice, this means we should expect to see more schematic, geometrical, and repetitive styles when societies and individuals are exposed to ongoing stress. At the same time, there is likely to be an ameliorative value in the identification of simple hidden variables standing behind otherwise chaotic experiences, given that these can provide a focus for ritual or 
therapeutic behaviors. Against this, more ornate, baroque, or organic styles should become visible when the producers of style exist in a stable, predictable milieu. In this regard, it has already been hypothesized that the impulse towards abstraction comes from 'the immense spiritual dread of space,' which leaves humanity 'tormented by the entangled inter-relationship and flux of the phenomena of the outer world' (Worringer, 1997, pp. 15-16). This is most obvious with respect to the linear, schematic exercises in artistic creation from the earliest parts of human history (Henshilwood et al., 2002), but it is no less true that the sensory richness of French impressionism and Victorian realism in the latter half of the nineteenth century could not contrast more with the revolutionary formalism of Russian art in the early twentieth century. And tellingly enough, the consolidation of the USSR and the regularization of daily life brought with it a migration to a less abstract, more realistic aesthetic. Nevertheless, I readily acknowledge that these claims-which take in the entirety of the cultural record-will need more evidence than I am able to supply here if they are to be compelling.

The second prediction is no less speculative, but hopefully equally provocative. This is the hypothesis that lower entropy styles should attend subject matter that is distant or cognitively remote. Here, the reasoning is that remote subject matter will, all things being equal, offer fewer familiar perceptual elements, and therefore make bigger claims on cognitive budget by requiring such elements to be explicitly memorized or inferred. What this means in practice is that any associated style is likely to make do with fewer, more schematic elements that are often repeated, as opposed to reproducing a familiar reality with a more expressive style. Once more, evidence for such a hypothesis requires a survey of the cultural record that I cannot offer, except to note that the fantastical cultural forms like myth, folktale, and the epic consistently make use of formulaic patterns, stock phrases, and predictable characters (Gray, 1971), just as genres like contemporary science fiction and fantasy are well-known for complementing far-reaching speculation with prosaic (i.e. predictable) delivery (Stockwell, 2014). However, a useful independent body of evidence in support of my view comes from construal level theory (CLT), a branch of social psychology that deals with the interaction between abstraction and psychological distance. The core discovery of CLT is that abstract representations cue expectations of psychological distance in the dimensions of space, time, likelihood, and social familiarity, just as the experience of psychological distance cues expectations of abstraction, with a similar relationship holding true for concreteness and nearness (Liberman \& Trope, 2009; Trope \& Liberman, 2010). In other words, CLT shows that cognition centering on far away, temporally distant, socially alien, or implausible themes is biased towards abstract modes of representation, with the reverse being true for representations that are near in those dimensions. I have explored this claim already with respect to science fiction in Carney (2017), where I show that four of its core topics-space travel, time travel, non-human agency, and counterfactual history-pair extremes of psychological distance with various forms of linguistic and cognitive abstraction. Extending this claim to other stylistic modes and cultural genres is certainly possible; doing so would both supply CLT with an 
explanatory mechanism for its claims and allow for these claims (and the associated prediction I am offering here) to be tested against rich cultural data.

The information-theoretical model of style therefore presents two clear further lines of development for future work. Here, my purpose will have been achieved if I have made clearer the precise ways in which anxiety about the future is alleviated by the injection of stylistic elements into the environment. Style, on this view, is less a cultural incidental or a hedonic decoration than it is the material implementation of perhaps the signature psycho-perceptual strategy of all forms of organic life (Friston, 2012; Friston et al., 2012). This is an ambitious claim, and even if true, a peremptory one. Nevertheless, the increasingly necessary intellectual project of reconciling our models of biological, cognitive, and cultural processes is an argument for at least pursuing it. If successful, the present chapter will have made one stepeven if only a negative one-towards doing so.

\section{References}

Adorno, T. W. (2004). Aesthetic theory. London, England: Continuum.

Alpers, S. (1987). Style is what you make it: The visual arts once again. In B. Lang (Ed.), The concept of style (pp. 137-162). Ithaca, NY: Cornell University Press.

Bach-y-Rita, P., \& Kercel, S. W. (2003). Sensory substitution and the human-machine interface. Trends in Cognitive Sciences, 7, 541-546. https://doi.org/10.1016/j.tics.2003.10.013

Barthes, R. (2000). Myth today. In S. Sontag (Ed.), A Roland Barthes Reader (pp. 93-149). London, UK: Vintage Books.

Botero, C. A., Pen, I., Komdeur, J., \& Weissing, F. J. (2010). The evolution of individual variation in communication strategies. Evolution, 64, 3123-3133. https://doi. org/10.1111/j.1558-5646.2010.01065.x

Bregman, L. M. (1967). The relaxation method of finding the common point of convex sets and its application to the solution of problems in convex programming. USSR Computational Mathematics and Mathematical Physics, 7(3), 200-217. https://doi. org/10.1016/0041-5553(67)90040-7

Brück, J. (2006). Death, exchange and reproduction in the British Bronze Age. European Journal of Archaeology, 9, 73-101. https://doi.org/10.1177/1461957107077707

Carney, J. (2017). The space between your ears: Construal level theory, cognitive science, and science fiction. In M. Burke \& E. T. Troscianko (Eds.), Cognitive literary science: Dialogues between literature and cognition (pp. 73-92). New York, NY: Oxford University Press.

Carney, J. (2019). Necessary fictions: Supernormal cues, complex cognition, and the nature of fictional narrative. In M. Grishakova \& M. Poulaki (Eds.), Narrative complexity: Cognition, embodiment, evolution (pp. 391-413). Lincoln, NE: University of Nebraska Press.

Carney, J., Robertson, C., \& Dávid-Barrett, T. (2019). Fictional narrative as a variational Bayesian method for estimating social dispositions in large groups. Journal of Mathematical Psychology, 93, 102279. https://doi.org/10.1016/j.jmp.2019.102279

Clark, A. (2016). Surfing uncertainty: Prediction, action, and the embodied mind. Oxford, UK: Oxford University Press.

Conrads, U. (1970). Programs and manifestoes on 20th-century architecture. Cambridge, MA: MIT Press.

Cover, T. M., \& Thomas, J. A. (2005). Elements of information theory (2nd ed.). Hoboken, NJ: Wiley. https://doi.org/10.1002/047174882X 
Craske, M. G. (2003). Origins of phobias and anxiety disorders: Why more women than men? Oxford, UK: Elsevier.

Craske, M. G., Rauch, S. L., Ursano, R., Prenoveau, J., Pine, D. S., \& Zinbarg, R. E. (2009). What is an anxiety disorder? Depression and Anxiety, 26, 1066-1085. https://doi.org/10.1002/ da. 20633

Curry, N. A., \& Kasser, T. (2005). Can coloring mandalas reduce anxiety? Journal of the American Art Therapy Association, 22, 81-85. https://doi.org/10.1080/07421656.2005.10129441

Danchev, A. (2011). 100 artists' manifestos: From the futurists to the stuckists. London, England: Penguin.

Dawkins, R., \& Krebs, J. R. (1979). Arms races between and within species. Proceedings of the Royal Society B, 205, 489-511. https://doi.org/10.1098/rspb.1979.0081

Dunbar, R. I. M., Lehmann, J., Korstjens, A. H., \& Gowlett, J. A. J. (2014). The road to modern humans: Time budgets, fission-fusion sociality, kinship and the division of labour in Hominin evolution. In R. I. M. Dunbar, C. Gamble, \& J. A. J. Gowlett (Eds.), Lucy to language: The benchmark papers. https://doi.org/10.1093/acprof:osobl/9780199652594.003.0015

Durt, C. (2017). Consciousness, culture, and significance. In C. Durt, T. Fuchs, \& C. Tewes (Eds.), Embodiment, enaction, and culture: Investigating the constitution of the shared world (pp. 65-86). Cambridge, MA: MIT Press.

Dutton, D. (2009). The art instinct: Beauty, pleasure, and human evolution. Oxford, UK: Oxford University Press.

Eco, U. (1985). Innovation and repetition: Between modern and post-modern aesthetics. Daedalus, 114(4), 161-184. Retrieved from http://www.jstor.org/stable/20025015

Ellsberg, D. (1961). Risk, ambiguity, and the savage axioms. The Quarterly Journal of Economics, 75, 643-669. https://doi.org/10.2307/1884324

Friston, K. (2009). The free-energy principle: A rough guide to the brain? Trends in Cognitive Sciences, 13, 293-301. https://doi.org/10.1016/j.tics.2009.04.005

Friston, K. (2012). A free energy principle for biological systems. Entropy, 14, 2100-2121. https:// doi.org/10.3390/e14112100

Friston, K. J., Shiner, T., FitzGerald, T., Galea, J. M., Adams, R., Brown, H., ... Bestmann, S. (2012). Dopamine, affordance and active inference. PLoS Computational Biology, 8(1), e1002327. https://doi.org/10.1371/journal.pcbi.1002327

Gallagher, S. (2008). Direct perception in the intersubjective context. Consciousness and Cognition, 17, 535-543. https://doi.org/10.1016/j.concog.2008.03.003

Gallagher, S. (2011). Narrative competence and the massive hermeneutical background. In P. Fairfield (Ed.), Education, dialogue and hermeneutics (pp. 21-38). London, England: Continuum.

Gatys, L. A., Ecker, A. S., \& Bethge, M. (2015). A neural algorithm of artistic style. CoRR, abs/1508.0. Retrieved from http://arxiv.org/abs/1508.06576

Georgeon, O. L., Marshall, J. B., \& Manzotti, R. (2013). ECA: An enactivist cognitive architecture based on sensorimotor modeling. Biologically Inspired Cognitive Architectures, 6, 46-57. https://doi.org/10.1016/j.bica.2013.05.006

Gray, B. (1971). Repetition in oral literature. The Journal of American Folklore, 84(333), 289-303. https://doi.org/10.2307/539805

Grillon, C., Baas, J. P., Lissek, S., Smith, K., \& Milstein, J. (2004). Anxious responses to predictable and unpredictable aversive events. Behavioral Neuroscience, 118, 916-924. https://doi. org/10.1037/0735-7044.118.5.916

Gwern. (2019). GPT-2 Neural Network Poetry. Retrieved August 21, 2019, from https://www. gwern.net/GPT-2

Hebidge, D. (1979). Subculture: The meaning of style. London, UK: Methuen.

Henshilwood, C. S., d'Errico, F., Yates, R., Jacobs, Z., Tribolo, C., Duller, G. A. T., ... Wintle, A. G. (2002). Emergence of modern human behavior: Middle Stone Age engravings from South Africa. Science, 295(5558), 1278-1280. Retrieved from http://science.sciencemag.org/ content/295/5558/1278.abstract 
Herry, C., Bach, D. R., Esposito, F., Di Salle, F., Perrig, W. J., Scheffler, K., ... Seifritz, E. (2007). Processing of temporal unpredictability in human and animal amygdala. Journal of Neuroscience, 27(22), 5958-5966. https://doi.org/10.1523/JNEUROSCI.5218-06.2007

Hirsh, J. B., Mar, R. A., \& Peterson, J. B. (2012). Psychological entropy: A framework for understanding uncertainty-related anxiety. Psychological Review, 119, 304-320. https://doi. org/10.1037/a0026767

Hunter, C. R., \& Pisoni, D. B. (2018). Extrinsic cognitive load impairs spoken word recognition in high- and low-predictability sentences. Ear and Hearing, 39, 378-389. https://doi.org/10.1097/ AUD.0000000000000493

Huntsman, P. (2016). Thinking about art: A thematic guide to art history. Chichester, UK: Wiley-Blackwell.

Hutto, D. (2009). Folk psychology and narrative practice. Journal of Consciousness Studies, 16(6-8), 9-39. Retrieved from http://scholar.google.com/scholar?hl=en\&btnG=Search\&q=in title:Folk+Psychology+as+Narrative+Practice\#0

Ishizu, T., \& Zeki, S. (2013). The brain's specialized systems for aesthetic and perceptual judgment. European Journal of Neuroscience, 37, 1413-1420. https://doi.org/10.1111/ejn.12135

Jameson, F. (1972). Marxism and form: Twentieth-century dialectical theories of literature. Princeton, NJ: Princeton University Press.

Jang, S.-H., Kang, S.-Y., Lee, H.-J., \& Lee, S.-Y. (2016). Beneficial effect of mindfulness-based art therapy in patients with breast cancer-A randomized controlled trial. EXPLORE, 12, 333-340. https://doi.org/10.1016/j.explore.2016.06.003

Kolchinsky, E. I., Kutschera, U., Hossfeld, U., \& Levit, G. S. (2017). Russia's new Lysenkoism. Current Biology, 27, R1042-R1047. https://doi.org/10.1016/j.cub.2017.07.045

Kullback, S., \& Leibler, R. A. (1951). On information and sufficiency. The Annals of Mathematical Statistics, 22, 79-86. Retrieved from http://www.jstor.org/stable/2236703

Kuzemsky, A. L. (2017). Statistical mechanics and the physics of many-particle model systems. Singapore: World Scientific Publishing Co Pte Ltd. https://doi.org/10.1142/10169

Lake, J. I., \& LaBar, K. S. (2011). Unpredictability and uncertainty in anxiety: A new direction for emotional timing research. Frontiers in Integrative Neuroscience, 5, 55. https://doi. org/10.3389/fnint.2011.00055

Lawson, R. P., Rees, G., \& Friston, K. J. (2014). An aberrant precision account of autism. Frontiers in Human Neuroscience, 8, 302. https://doi.org/10.3389/fnhum.2014.00302

Liberman, N., \& Trope, Y. (2009). The psychology of transcending the here and now. Science, 322(11), 1201-1206. https://doi.org/10.1126/science.1161958

Lloyd, P. B. (2017). From modernism to metro maps: Mondrian, Beck, and Salomon. Brief Encounters, 1. https://doi.org/10.24134/be.v1i1.38

Marr, D. (1982). Vision. New York, NY: W.H. Freeman.

Marr, D. (2010). Vision: A computational investigation into the human representation and processing of visual information. Cambridge, MA: MIT Press.

Martindale, C. (1990). The clockwork muse: The predictability of artistic change. New York, NY: Basic Books.

Martindale, C., \& Locher, P. (2009). The evolution and end of art as Hegelian tragedy. Empirical Studies of the Arts, 27, 133-140. https://doi.org/10.2190/EM.27.2.c

Meyer, L. B. (1987). Toward a theory of style. In B. Lang (Ed.), The concept of style (pp. 21-71). Ithaca, NY: Cornell University Press.

Mithen, S. (1995). Palaeolithic archaeology and the evolution of mind. Journal of Archaeological Research, 3, 305-332. Retrieved from http://www.jstor.org/stable/41053107

Munro, T. (1946). Style in the arts: A method of stylistic analysis. The Journal of Aesthetics and Art Criticism, 5, 128-158. https://doi.org/10.2307/425801

Nakayama, R., Motoyoshi, I., \& Sato, T. (2018). Discretized theta-rhythm perception revealed by moving stimuli. Scientific Reports, 8(1), 5682. https://doi.org/10.1038/s41598-018-24131-6

Nietzsche, F. W. (1927). The birth of tragedy (C. P. Fadiman, Trans.). New York, NY: Modern Library. 
Peirce, C. S. (1958). Collected papers of Charles Sanders Peirce. Cambridge, MA: Harvard University Press.

Pigeaud, R. (2007). Determining style in Palaeolithic cave art: A new method derived from horse images. Antiquity, 81(312), 409-422. https://doi.org/10.1017/S0003598X00095272

Pinker, S. (2007). Toward a consilent study of literature. Philosophy and Literature, 31, 161-177. https://doi.org/10.1353/phl.2007.0016

Sandmire, D. A., Gorham, S. R., Rankin, N. E., \& Grimm, D. R. (2012). The influence of art making on anxiety: A pilot study. Art Therapy, 29, 68-73. https://doi.org/10.1080/0742165 6.2012 .683748

Schapiro, M. (1997). Impressionism: Reflections and perceptions. New York, NY: George Braziller.

Schebesch, A. (2013). Five anthropomorphic figurines of the Upper Paleolithic-Communication through body language. Mitteilungen der Gesellschaft für Urgeschichte, 22, 61-100.

Seeley, W. P. (2013). Art, meaning, and perception: A question of methods for a cognitive neuroscience of art. British Journal of Aesthetics, 53, 443-460. https://doi.org/10.1093/aesthj/ayt022

Shannon, C. E. (1948). A mathematical theory of communication. The Bell System Technical Journal, 27(3), 379-423. https://doi.org/10.1145/584091.584093

Shklovsky, V. (1965). Art as technique. In L. Lemon \& M. Reiss (Trans.), Russian formalist criticism: Four essays (pp. 3-24). Lincoln, NE: University of Nebraska Press.

Sims, C. R. (2016). Rate-distortion theory and human perception. Cognition, 152, 181-198. https://doi.org/10.1016/j.cognition.2016.03.020

Sinha, P., Kjelgaard, M. M., Gandhi, T. K., Tsourides, K., Cardinaux, A. L., Pantazis, D., ... Held, R. M. (2014). Autism as a disorder of prediction. Proceedings of the National Academy of Sciences of the United States of America, 111(42), 15220-15225. https://doi.org/10.1073/ pnas. 1416797111

Slayton, S. C., D'Archer, J., \& Kaplan, F. (2010). Outcome studies on the efficacy of art therapy: A review of findings. Art Therapy: Journal of the American Art Therapy Association, 27(3), $108-118$.

Smith, B. H. (1980). Narrative versions, narrative theories. Critical Inquiry, 7, 213-236. Retrieved from http://www.jstor.org/stable/1343185

Sørensen, M. L. S., \& Rebay, K. (2008). From substantial bodies to the substance of bodies: Analysis of the transition from inhumation to cremation during the Middle Bronze Age in Europe. In D. Broić \& J. Robb (Eds.), Past bodies: Body-centered research in archaeology (pp. 59-68). Oxford, UK: Oxbow Books.

Stockwell, P. (2014). The poetics of science fiction. Oxford, UK: Routledge.

Stout, D., Hecht, E., Khreisheh, N., Bradley, B., \& Chaminade, T. (2015). Cognitive demands of Lower Paleolithic toolmaking. PLoS One, 10(4), e0121804. https://doi.org/10.1371/journal. pone. 0121804

Sullivan, D. (2016). Cultural-existential psychology: The role of culture in suffering and threat. Cambridge, UK: Cambridge University Press.

Thom, R. (1990). Semio physics: A sketch (V. Meyer, Trans.). Amsterdam, Netherlands: Addison-Wesley.

Tooby, J., \& Cosmides, L. (2001). Does beauty build adapted minds? Toward an evolutionary theory of aesthetics, fiction and the arts. SubStance, 30(1), 6-27. https://doi.org/10.1353/ sub.2001.0017

Trope, Y., \& Liberman, N. (2010). Construal-level theory of psychological distance. Psychological Review, 117(2), 440-463. https://doi.org/10.1037/a0018963

van der Vennet, R., \& Serice, S. (2012). Can coloring mandalas reduce anxiety? A replication study. Art Therapy, 29, 87-92. https://doi.org/10.1080/07421656.2012.680047

Van Lier, H. N., \& Steiner, F. R. (1982). A review of the Zuiderzee reclamation works: An example of Dutch physical planning. Landscape Planning, 9, 35-59. https://doi. org/10.1016/0304-3924(82)90010-7

Wintle, M. (2000). An economic and social history of the Netherlands, 1800-1920: Demographic ... - Michael Wintle_Google Books. Cambridge, UK: Cambridge University Press. Retrieved 
from https://books.google.co.uk/books?id=EqRAAjR5XncC\&pg=PA245\&dq=Dutch+technol ogy+1920s\&hl=en\&sa=X\&ved=0ahUKEwiRgJyo_9zkAhUjpHEKHRzQA0IQ6AEIKjAA\#v $=$ onepage $\& \mathrm{q}=$ Dutch+technology $+1920 \mathrm{~s} \& \mathrm{f}=$ false

Worringer, W. (1997). Abstraction and empathy: A contribution to the psychology of style. Chicago, IL: Ivan R. Dee.

Open Access This chapter is licensed under the terms of the Creative Commons Attribution 4.0 International License (http://creativecommons.org/licenses/by/4.0/), which permits use, sharing, adaptation, distribution and reproduction in any medium or format, as long as you give appropriate credit to the original author(s) and the source, provide a link to the Creative Commons license and indicate if changes were made.

The images or other third party material in this chapter are included in the chapter's Creative Commons license, unless indicated otherwise in a credit line to the material. If material is not included in the chapter's Creative Commons license and your intended use is not permitted by statutory regulation or exceeds the permitted use, you will need to obtain permission directly from the copyright holder.

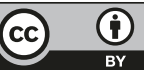

